\section{Cureus}

Received 06/25/2018

Review began 07/03/2018

Review ended 07/10/2018

Published 07/13/2018

\section{(c) Copyright 2018}

Klebanov et al. This is an open access article distributed under the terms of the Creative Commons Attribution License CC-BY 3.0., which permits unrestricted use, distribution, and reproduction in any medium, provided the original author and source are credited.

\title{
Pleomorphic Dermal Sarcoma of the Scalp
}

\author{
Nikolai Klebanov $^{1}$, Mai P. Hoang ${ }^{2}$, Bobby Y. Reddy ${ }^{1}$ \\ 1. Dermatology, Massachusetts General Hospital, Boston, USA 2. Pathology, Massachusetts General \\ Hospital/Harvard Medical School, Boston, USA
}

$\square$ Corresponding author: Nikolai Klebanov, nikolai.klebanov@gmail.com Disclosures can be found in Additional Information at the end of the article

\section{Abstract}

Pleomorphic dermal sarcoma (PDS) is a rare mesenchymal tissue tumor. Distinguishing PDS from similar conditions, such as atypical fibroxanthoma (AFX), its less aggressive tumor counterpart, is difficult, as they are clinically and histologically similar. We present a case of a 77-year-old man presenting with a large nodular scalp lesion of three weeks duration.

Pathology revealed a $3.3 \mathrm{~cm}$ invasive pleomorphic dermal sarcoma. Surgical excision with $2 \mathrm{~cm}$ margins was performed with successful healing of the graft. This case highlights a rare case of a large pleomorphic dermal sarcoma and discusses the histological features and management of PDS.

Categories: Dermatology, Pathology, Oncology

Keywords: undifferentiated pleomorphic sarcoma, pleomorphic dermal sarcoma, atypical fibroxanthoma

\section{Introduction}

Atypical fibroxanthoma (AFX) and pleomorphic dermal sarcoma (PDS), formerly known as malignant fibrous histiocytoma (MFH), are rare mesenchymal tissue tumors. These tumors are clinically and morphologically similar, and it is debated whether PDS represents a separate clinical entity from AFX or it is merely a more aggressive variant. AFX occurs more frequently than PDS; however, both of these entities are quite uncommon. AFX cases have been moderately well-documented, thus allowing for a study of the risk factors and the genetics of these mesenchymal malignancies.

AFX tumors affect elderly patients (usually male patients between the ages of 70 and 80) [1-2], favor ultraviolet (UV)-damaged skin of the head and neck, and are associated with several known risk factors, including radiation (including UV), xeroderma pigmentosum, and a history of organ transplantation [3-8]. On histological examination, AFX lesions are characterized by pleomorphic tumor cells, atypical spindle cells, and giant cells. The genetic underpinnings of AFX are poorly understood given the rarity of these tumors. Case series have identified UVsignature mutations in the TP53 gene [9-10] as well as likely oncogenic HRAS, KRAS, NOTCH1/2, and FAT1 mutations [7,11] by the sequencing of AFX tumor samples. Given the predilection of AFX for the sun-exposed skin of the head and neck in older patients and the presence of UV-signature p53 mutations, AFX emerges as a tumor with similar characteristics to non-melanoma skin cancers, such as basal cell carcinoma (BCC) and squamous cell carcinoma (SCC) (which are currently thought to be attributable to cumulative and chronic sun exposure). In fact, a personal history of BCC or SCC is frequently identified in patients presenting with AFX [12-13].

Distinguishing AFX from PDS is vital, given that PDS is a much more clinically aggressive 


\section{Cureus}

tumor, requiring prompt diagnosis and decisive treatment. However, clinically distinguishing the two cutaneous diseases is often difficult because of the similarities in their gross and microscopic morphologies. Several studies support the treatment of AFX with Mohs surgery [12-15] but less is known about the treatment of the more aggressive PDS variant.

We present a case of a mesenchymal tissue tumor affecting the scalp of an elderly male patient. We discuss the pathological diagnosis of the patient's tumor and the successful surgical management of the lesion. Our patient's case illustrates the rare pleomorphic dermal sarcoma malignancy. This case may help clinicians be more aware of the related atypical fibroxanthoma (AFX) and pleomorphic dermal sarcoma (PDS) mesenchymal tumors and of the surgical management that can be used for the management of these rare conditions.

\section{Case Presentation}

A 77-year-old man with no history of skin cancer presented to the outpatient dermatology clinic for a scalp lesion of three weeks duration. He reported rapid lesion growth, but no change in the overall appearance nor any associated symptoms. Examination revealed a mobile, round, exophytic nodule with overlying ulceration and hemorrhagic crust, approximately $1.5 \mathrm{~cm}$ in diameter (Figure 1A). Given a high clinical suspicion of malignancy, the lesion was excised three weeks following the initial visit using a fusiform (elliptical) incision with $1 \mathrm{~cm}$ margins. The lesion immediately prior to the procedure is seen in Figure $1 B$.
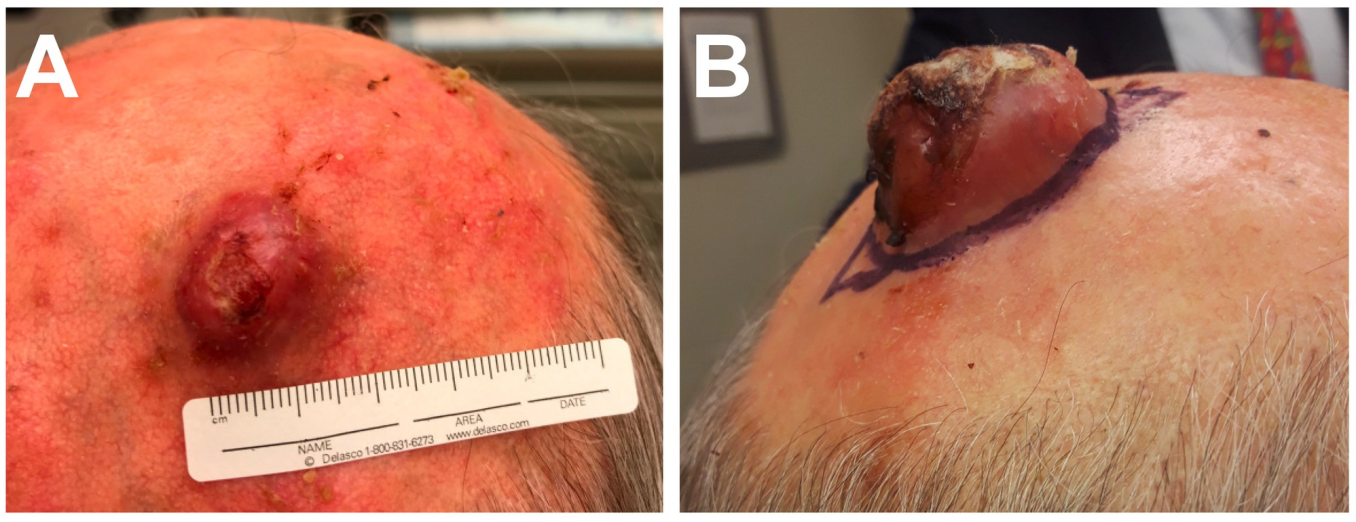

\section{FIGURE 1: Gross scalp lesion morphology}

1A. Cutaneous $1.5 \mathrm{~cm}$ nodule at initial visit three weeks after the patient initially noted the lesion. 1B. Erythematous ulcerated nodule prior to the wide, local excision on the third week following initial clinic presentation.

A histopathologic examination of an excisional biopsy of the cutaneous lesion revealed a proliferation of spindle and pleomorphic tumor cells, which flattened the overlying epidermis (Figure 2A) and extended to the subcutaneous tissue (Figure 2B). The tumor cells were large and polygonal and contained eosinophilic cytoplasm. They expressed diffuse CD10 positivity (Figure 2C) and focal CD68 positivity. The tumor cells were negative for $\mathrm{p} 40$ and SOX10, excluding the possibility of sarcomatoid carcinoma and melanoma, respectively. The histopathologic findings were found to be consistent with invasive pleomorphic dermal sarcoma. The tumor measured $3.3 \mathrm{~cm}$ at the largest dimension, and tumor cells were found to be present at the surgical margin. There was no evidence of lymphovascular or perineural invasion. 


\section{Cureus}
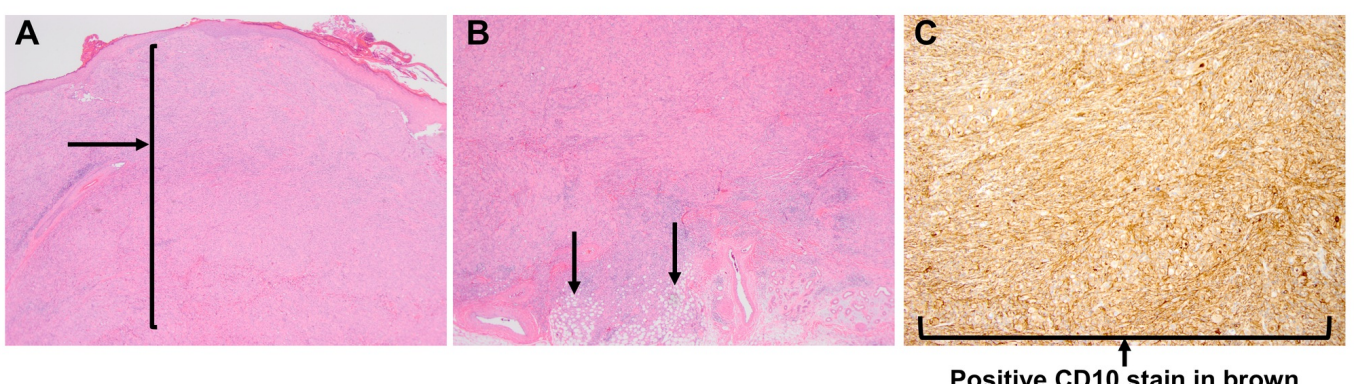

\section{FIGURE 2: Histological tumor features}

2A. An expansile tumor in the dermis (original magnification $\mathrm{X} 40$ ). 2B: Involvement of subcutaneous fat is noted (original magnification X40). 2C: Diffuse CD10 expression by the tumor cells (original magnification X100).

A surgical, wide, local excision was planned. Preparatory computed tomography (CT) imaging revealed an approximately $3.9 \mathrm{~cm}$ focus of enhancement with central ulceration in the soft tissue of the posterior scalp and confirmed a lack of bony erosion in the underlying calvarium. No metastatic adenopathy was appreciated. Figure $3 \mathrm{~A}$ demonstrates the scalp vertex prior to surgery. A wide local excision was performed using a $2 \mathrm{~cm}$ margin around the remaining tumor for a $7.5 \mathrm{~cm}$ area of planned excision. The repair utilized a $7 \times 3.5 \mathrm{~cm}$ full-thickness skin graft from the left upper chest. The immediate post-surgical course was uncomplicated. Wound care with sterile petrolatum gauze dressing (Figure 3B) sutured to the scalp for two weeks helped with successful graft healing (Figure 3C). At the one-month follow-up after the operation, a review of systems did not reveal systemic relapse and an examination of the surgical scar and cervical lymph nodes did not reveal local recurrence. The patient will continue to have close monitoring by the dermatology team twice per year.
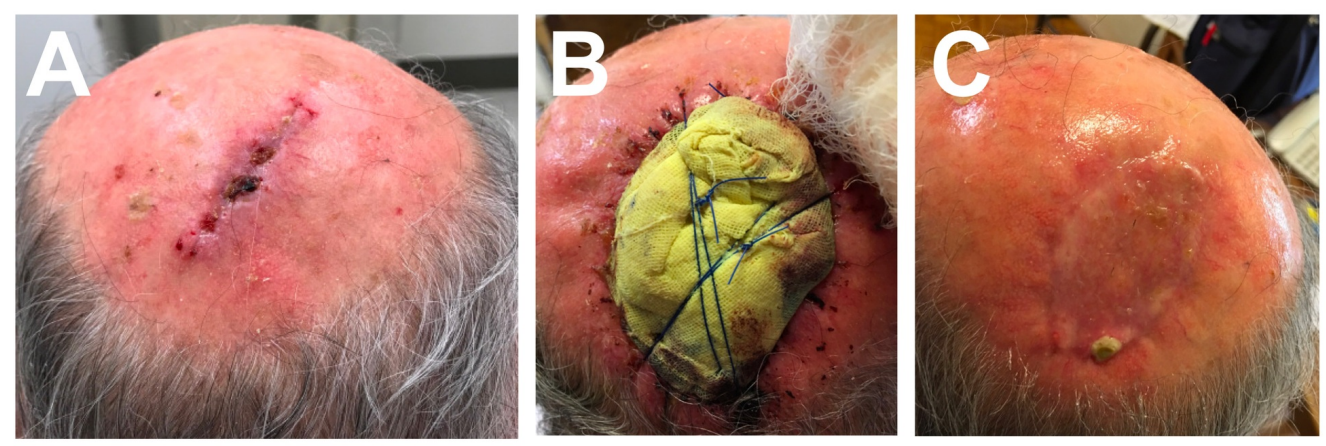

\section{FIGURE 3: Outcomes of surgical lesion management}

3A. Surgical wound two weeks following initial narrow elliptical excision. 3B. Petrolatum gauze scalp dressing applied following the excision with wide $(2 \mathrm{~cm})$ surgical margins. 3C. Healthy fullthickness skin graft healing two weeks following the wide, local excision.

\section{Discussion}

Given the high risk of malignant behavior in pleomorphic dermal sarcoma (PDS) tumors, it is important to distinguish PDS from its more benign atypical fibroxanthoma (AFX) counterpart. This decision is made difficult by the morphological similarities between AFX and PDS, such as 
spindle or round cell histology, the presence of pleomorphism and atypical mitotic figures, and CD10 marker positivity. PDS sometimes reveals more aggressive histological features than AFX, such as evidence of deeper subdermal involvement, the presence of lymphovascular or perineural invasion, and/or necrosis [7].

In the case presented here, the histological examination did not reveal any evidence of lymphovascular or perineural invasion. Likewise, as diffuse CD10 positivity is observed in both AFX and PDS tumors, CD10 positivity alone could not be used to make the diagnosis of PDS. However, the involvement of the deep subcutis favors a diagnosis of PDS [3] and given the gross size of the tumor and the depth of penetration involving the subcutaneous fat (Figures 2A-2B), there was a low threshold to treat this growth aggressively as a pleomorphic dermal sarcoma. In the literature, reported treatments for atypical fibroxanthoma (AFX) include cryotherapy, radiation, and surgical methods: wide local excision and Mohs micrographic surgery. Surgical excision is preferred over cryotherapy and irradiation. Cryotherapy is not recommended due to the risk of recurrence or metastasis, and irradiation poses the risk of causing tumor DNA dysregulation, resulting in progression to a more aggressive tumor [16]. A meta-analysis of 23 studies comparing treatment with Mohs micrographic surgery to excision for atypical fibroxanthoma suggested that Mohs surgery was associated with a lower recurrence rate than wide local excision [15].

Less is known overall regarding PDS management given the tumor's rarity. Complete surgical excision is considered as the first line option for PDS management [17], and an incomplete excision places the patient at increased risk of local recurrence [18-19], with the study by Tardío et al. reporting a $20 \%$ rate of local recurrence after surgical management, all in patients with incomplete resections. Although the metastatic potential of this rare tumor is estimated to be lower than 5\% [19-20], given the poor prognosis of untreated PDS, it is vital for clinicians to be aware of this tumor and proceed to prompt surgical management, ensuring clear margins.

\section{Conclusions}

Pleomorphic dermal sarcoma is a rare tumor affecting mesenchymal tissue. We present the case of pleomorphic dermal sarcoma occurring on the scalp of a 77-year-old male patient. Successful treatment with a wide, local excision was performed. This case highlights the morphologic and histologic characteristics of PDS and discusses what is known about the common treatments of rare mesenchymal malignancies such as atypical fibroxanthoma (AFX) and PDS.

\section{Additional Information \\ Disclosures}

Human subjects: Consent was obtained by all participants in this study. Conflicts of interest: In compliance with the ICMJE uniform disclosure form, all authors declare the following:

Payment/services info: All authors have declared that no financial support was received from any organization for the submitted work. Financial relationships: All authors have declared that they have no financial relationships at present or within the previous three years with any organizations that might have an interest in the submitted work. Other relationships: All authors have declared that there are no other relationships or activities that could appear to have influenced the submitted work.

\section{References}

1. Ziemer M: Atypical fibroxanthoma. J Dtsch Dermatol Ges. 2012, 10:537-550. 10.1111/j.16100387.2012.07980.x

2. Wollina U, Schonlebe J, Ziemer M, et al.: Atypical fibroxanthoma: a series of 56 tumors and an unexplained uneven distribution of cases in southeast Germany. Head Neck. 2015, 37:829- 
834. 10.1002/hed.23673

3. Iorizzo LJ, Brown MD: Atypical fibroxanthoma: a review of the literature . Dermatol Surg. 2011, 37:146-157. 10.1111/j.1524-4725.2010.01843.x

4. Gru AA, Santa Cruz DJ: Atypical fibroxanthoma: a selective review . Semin Diagn Pathol. 2013, 30:4-12. 10.1053/j.semdp.2012.01.001

5. McCalmont TH: AFX: what we now know. J Cutan Pathol. 2011, 38:853-856. 10.1111/j.16000560.2011.01802.X

6. Mentzel T, Requena L, Brenn T: Atypical fibroxanthoma revisited. Surg Pathol Clin. 2017, 10:319-335. 10.1016/j.path.2017.01.007

7. Griewank KG, Wiesner T, Murali R, et al.: Atypical fibroxanthoma and pleomorphic dermal sarcoma harbor frequent NOTCH1/2 and FAT1 mutations and similar DNA copy number alteration profiles. Mod Pathol. 2018, 31:418-428. 10.1038/modpathol.2017.146

8. McCoppin HH, Christiansen D, Stasko T, Washington C, Martinez JC, Brown MD, Zwald FO: Clinical spectrum of atypical fibroxanthoma and undifferentiated pleomorphic sarcoma in solid organ transplant recipients: a collective experience. Dermatol Surg. 2012, 38:230-239. 10.1111/j.1524-4725.2011.02180.x

9. Dei Tos AP, Maestro R, Doglioni C, Gasparotto D, Boiocchi M, Laurino L, Fletcher CD: Ultraviolet-induced p53 mutations in atypical fibroxanthoma . Am J Pathol. 1994, 145:11-17.

10. Sakamoto A, Oda Y, Itakura E, Oshiro Y, Nikaido O, Iwamoto Y, Tsuneyoshi M: Immunoexpression of ultraviolet photoproducts and p53 mutation analysis in atypical fibroxanthoma and superficial malignant fibrous histiocytoma. Mod Pathol. 2001, 14:581-588. 10.1038/modpathol.3880354

11. Sakamoto A, Oda Y, Itakura E, et al.: $\mathrm{H}-, \mathrm{K}-$, and $\mathrm{N}$-ras gene mutation in atypical fibroxanthoma and malignant fibrous histiocytoma. Hum Pathol. 2001, 32:1225-1231. 10.1053/hupa.2001.28953

12. Ang GC, Roenigk RK, Otley CC, Kim Phillips P, Weaver AL: More than 2 decades of treating atypical fibroxanthoma at mayo clinic: what have we learned from 91 patients?. Dermatol Surg. 2009, 35:765-772. 10.1111/j.1524-4725.2009.01126.x

13. Seavolt M, McCall M: Atypical fibroxanthoma: review of the literature and summary of 13 patients treated with Mohs micrographic surgery. Dermatol Surg. 2006, 32:435-441. 10.1111/j.1524-4725.2006.32087.x

14. Limmer BL, Clark DP: Cutaneous micrographic surgery for atypical fibroxanthoma. Dermatol Surg. 1997, 23:553-557.

15. Tolkachjov SN, Kelley BF, Alahdab F, Erwin PJ, Brewer JD: Atypical fibroxanthoma: systematic review and meta-analysis of treatment with Mohs micrographic surgery or excision. J Am Acad Dermatol. 2018, 10.1016/j.jaad.2018.06.048

16. Love WE, Schmitt AR, Bordeaux JS: Management of unusual cutaneous malignancies: atypical fibroxanthoma, malignant fibrous histiocytoma, sebaceous carcinoma, extramammary Paget disease. Dermatol Clin. 2011, 29:201-216. 10.1016/j.det.2011.02.007

17. Kim JI, Choi YJ, Seo HM, et al.: Case of pleomorphic dermal sarcoma of the eyelid treated with micrographic surgery and secondary intention healing. Ann Dermatol. 2016, 28:632-636. 10.5021/ad.2016.28.5.632

18. Miller K, Goodlad JR, Brenn T: Pleomorphic dermal sarcoma: adverse histologic features predict aggressive behavior and allow distinction from atypical fibroxanthoma. Am J Surg Pathol. 2012, 36:1317-1326. 10.1097/PAS.0b013e31825359e1

19. Tardio JC, Pinedo F, Aramburu JA, Suarez-Massa D, Pampin A, Requena L, Santonja C: Pleomorphic dermal sarcoma: a more aggressive neoplasm than previously estimated . J Cutan Pathol. 2016, 43:101-112. 10.1111/cup.12603

20. Sharma SR, Meligonis G, Todd P: Uncommon skin cancer: pleomorphic dermal sarcoma . BMJ Case Rep. 2018, 10.1136/bcr-2018-224483 